Citation: E.A. Rangel-Montoya, M. Paolinelli, P.E. Rolshausen, C. Valenzuela-Solano, R. Hernandez-Martinez (2021) Characterization of Lasiodiplodia species associated with grapevines in Mexico. Phytopathologia Mediterranea 60(2): 237-251. doi: 10.36253/ phyto-12576

Accepted: April 11, 2021

Published: September 13, 2021

Copyright:@2021 E.A. Rangel-Montoya, M. Paolinelli, P.E. Rolshausen, C. Valenzuela-Solano, R. Hernandez-Martinez. This is an open access, peerreviewed article published by Firenze University Press (http://www.fupress. $\mathrm{com} / \mathrm{pm}$ ) and distributed under the terms of the Creative Commons Attribution License, which permits unrestricted use, distribution, and reproduction in any medium, provided the original author and source are credited.

Data Availability Statement: All relevant data are within the paper and its Supporting Information files.

Competing Interests: The Author(s) declare(s) no conflict of interest.

Editor: José R. Úrbez Torres, Agriculture and Agri-Food Canada, Summerland, British Columbia, Canada.

\section{Research Papers \\ Characterization of Lasiodiplodia species associated with grapevines in Mexico}

\author{
Edelweiss A. RANGEL-MONTOYA ${ }^{1}$, Marcos PAOLINELli ${ }^{2,3}$, Philippe E. \\ ROLSHAUSEN $^{4}$, CESAR VALENZUELA-SOLANO ${ }^{5}$, RUFINA HERNANDEZ- \\ MARTINEZ ${ }^{1, *}$ \\ ${ }^{1}$ Departamento de Microbiología, Centro de Investigación Científica y de Educación \\ Superior de Ensenada (CICESE), Ensenada, Baja California, 22860, Mexico \\ ${ }^{2}$ Consejo Nacional de Investigaciones Científicas y Técnicas (CONICET) \\ ${ }^{3}$ Instituto Nacional de Tecnología Agropecuaria. Estación Experimental Agropecuaria \\ Mendoza INTA, Luján de Cuyo, Mendoza, 5534, Argentina \\ ${ }^{4}$ Department of Botany and Plant Sciences. University of California, Riverside, Califor- \\ nia, 92521, USA \\ ${ }^{5}$ Instituto Nacional de Investigaciones Forestales, Agrícolas y Pecuarias (INIFAP). Campo \\ Experimental Costa de Ensenada, Ensenada, Baja California, 22880, Mexico \\ ${ }^{*}$ Corresponding author. E-mail: ruhernan@cicese.mx
}

Summary. Botryosphaeria dieback is one of the most prevalent grapevine trunk diseases (GTDs), and is caused by fungi in the Botryosphaeriaceae. Fungi invade grapevine vascular systems mainly through pruning wounds, and cause cankers and necrotic lesions, which lead to grapevine decline and death. Lasiodiplodia theobromae has been reported as a highly virulent pathogen of grapevine, and was previously reported in Mexican vineyards. The taxonomy of Lasiodiplodia was recently revised, adding new species, and some were reduced to synonymy. This study aimed to characterize Lasiodiplodia producing grapevine dieback symptoms in Sonora and Baja California, Mexico. Using the phylogenetic markers tef1-a and ITS regions, Lasiodiplodia brasiliensis, L. crassispora, L. exigua, and L. gilanensis were identified. Lasidiplodia exigua was the most prevalent species. Lasiodiplodia brasiliensis and L. gilanensis were very virulent to 'Cabernet Sauvignon' plants, while L. exigua and L. gilanensis were less virulent, and $L$. crassispora did not produce lesions at 2 months post-inoculation. The optimum temperature of the Lasiodiplodia spp. was $28^{\circ} \mathrm{C}$, but all four species grew up to $37^{\circ} \mathrm{C}$, and the isolates of L. exigua grew slowly at $40^{\circ} \mathrm{C}$. This is the first report of the four of Lasiodiplodia species in vineyards of Mexico.

Keywords. Grapevine Trunk Diseases (GTDs), Botryosphaeria dieback, Botryosphaeriaceae.

\section{INTRODUCTION}

In Baja California and Sonora, Mexico, grapes are one of the most economically important fruit crops (García-Robles et al., 2007; GonzálezAndrade, 2015). Baja California produces close to $90 \%$ of Mexico's wines, while Sonora produces approx. 95\% of Mexican table grapes (SIAP, 2019). 
Botryosphaeria dieback is a degenerative wood disease caused by Botryosphaeriaceae fungi, this disease has cosmopolitan distribution and predominates in warm climate regions (Úrbez-Torres, 2011; Gramaje et al., 2018). Fungi in this family are known as opportunistic or latent plant pathogens, as they can remain endophytic for long periods in host tissues without causing symptoms (Slippers et al., 2007).

More than 30 species in the Botryosphaeriaceae have been associated with Botryosphaeria grapevine dieback, and these are in Botryosphaeria, Diplodia, Dothiorella, Lasiodiplodia, Neoscytalidium, Neofusicoccum, Sphaeropsis, and Spencermartinsia (Úrbez-Torres, 2011; Rolshausen et al., 2013; Stempien et al., 2017; Gramaje et al., 2018). The main symptoms caused by these fungi are vascular discolouration and perennial cankers in host plant vascular bundles, by occlusion of xylem and phloem, which leads to the death of branches and eventually of entire plants. This disease is distinguished from Eutypa dieback because it is not known to cause particular foliar symptoms (Úrbez-Torres, 2011; Bertsch et al., 2013; Billones-Baaijens and Savocchia, 2019). Species in the Botryosphaeriaceae were commonly found in grapevines 7 to 10 years old and older, mainly in plants where large pruning wounds had been made in vines (Gubler et al., 2005). However, incidence of symptoms caused by this group of fungi has greatly increased in recent years, especially in young vineyards (Gramaje and Armengol, 2011; Gispert et al., 2020).

Among the Botryosphaeriaceae, the Lasiodiplodia has been reported as highly virulent on grapevines (Úrbez-Torres and Gubler, 2009), and has also been identified on more than 500 host species (Punithalingam, 1976). Some of the main morphological characteristics of Lasiodiplodia include hyaline and smooth conidiogenous cells, with cylindrical to conical shapes, which produce conidia with subovoid to ellipsoid-ovoid shapes and which are hyaline without septa, or dark-brown with single septae (Phillips et al., 2013). Lasiodiplodia are globally distributed, mainly in the tropics and subtropics, and are probably spread when plants are transported between regions due to the lack of restrictions on the movement of propagation material (Cruywagen et al., 2017; Mehl et al., 2017). Lasiodiplodia theobromae is the type species of the genus (Alves et al., 2008), and this species is comprised of many cryptic species because of their morphological similarity (Alves et al., 2008; Mehl et al., 2017). As a result, the taxonomy of Lasiodiplodia has undergone revisions, and new species have been introduced (Dissanayake et al., 2016; Tibpromma et al., 2018). Several Lasiodiplodia species have been reduced to synonymy, particularly those with morphology similar to Lasiodiplodia mahajangana, L. plurivora and $L$. theobromae. There are currently 34 accepted Lasiodiplodia species (Zhang et al., 2021).

The only Lasiodiplodia species causing perennial cankers and dieback that has been reported in Mexican vineyards is $L$. theobromae (Úrbez-Torres et al., 2008). However, given the recent taxonomical revision of Lasiodiplodia, we hypothesize that the species diversity within that group is broader than initially reported. Hence, the present study aimed to clarify and update the taxonomy of Lasiodiplodia present in vineyards from Baja California and Sonora, Mexico, and to evaluate the pathogenicity of these fungi to grapevine.

\section{MATERIALS AND METHODS}

Fungal isolation and morphological characterization of Lasiodiplodia $s p$.

This study encompassed ten vineyards in the main grape-growing areas of the States of Baja California and Sonora, from which 35 samples from grapevines exhibiting Botryosphaeria dieback symptoms were taken from trunks and branches (Figure 1). Small pieces of symptomatic plant tissue were obtained from each diseased plant, and these were immersed in 95\% ethanol, quickly flamed, and then placed onto potato dextrose agar (PDA; Difco) supplemented with $25 \mathrm{mg} \mathrm{mL}^{-1}$ chloramphenicol in Petri plates. The plates were incubated at $30^{\circ} \mathrm{C}$ until fungal growth was observed. Smoke-gray fungal colonies with abundant aerial mycelium were sub-cultured onto PDA plates to obtain pure cultures, and were then preserved at $4^{\circ} \mathrm{C}$ in $20 \%$ glycerol.

Pure cultures were grown on PDA and incubated at $30^{\circ} \mathrm{C}$ for $7 \mathrm{~d}$ to determine morphological characteristics of fungal isolates, including their pigmentation and formation of aerial mycelium. Pycnidium production was induced using liquid Minimal Medium 9 (MM9) (10 g.L $\mathrm{L}^{-1}$ glucose, $1.0 \mathrm{~g} \cdot \mathrm{L}^{-1} \mathrm{NH}_{4} \mathrm{Cl}, 0.5 \mathrm{~g} \cdot \mathrm{L}^{-1} \mathrm{NaCl}, 2.5 \mathrm{~g} \cdot \mathrm{L}^{-1}$ $\left.\mathrm{K}_{2} \mathrm{HPO}_{4}, 2.5 \mathrm{~g} \cdot \mathrm{L}^{-1} \mathrm{KH}_{2} \mathrm{PO}_{4}\right)$ in flasks supplemented with sterile pine needles $(5 \% \mathrm{w} / \mathrm{v})$. The flasks were incubated at room temperature under an ultraviolet electromagnetic radiation lamp, using a $12 \mathrm{~h}$ light and $12 \mathrm{~h}$ darkness regime for $15 \mathrm{~d}$. Formed pycnidia were suspended in $0.5 \%$ Tween 20 to obtain conidia, which were observed under a light microscope (Nikon Eclipse E200). Images of the conidia were captured with an Infinity 1 Lumenera camera, and analyzed using Infinity Analyze v 6.5.4 and ImageJ software. To compare conidium size across species, one-way ANOVA followed by a post hoc Fisher LSD analysis $(\alpha<0.05)$ were carried on these data using STATISTICA 8.0. 

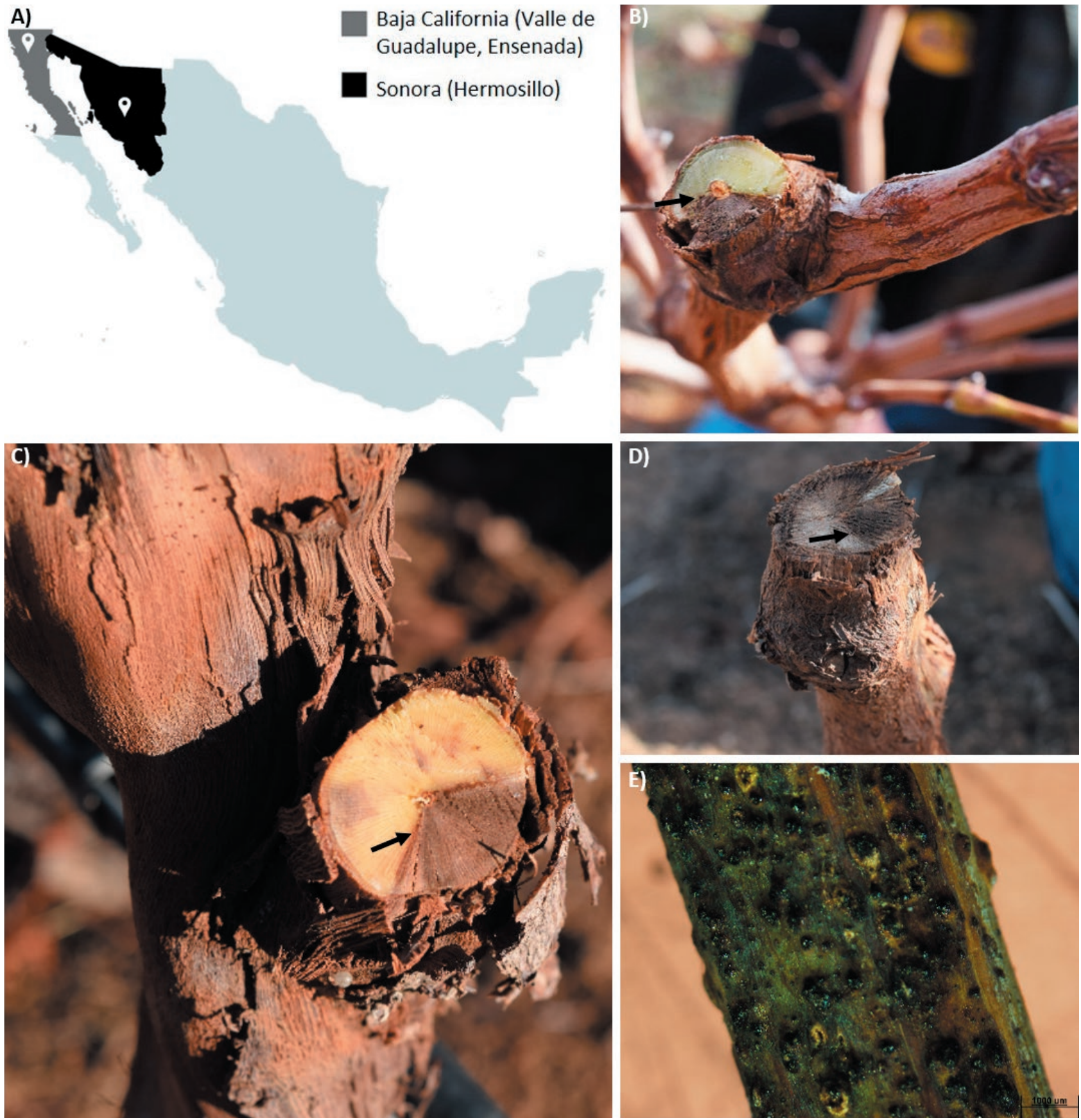

Figure 1. Locations of study sites and symptoms of Botryosphaeria dieback in Vitis vinifera associated with Lasiodiplodia spp. A) Field study sites in Baja California and Sonora regions. B-D) Grapevine plants showing vascular necroses, wedge-shape cankers and wood necroses. E) Pycnidia observed under a stereoscopic microscope found in some grapevine samples.

DNA extraction and PCR amplification from Lasiodiplodia spp. isolates

Total genomic DNA of each fungus isolate was extracted from mycelia recovered from cultures $(3 \mathrm{~d}$ in
$\mathrm{PDB}$ at $30^{\circ} \mathrm{C}$ ), using the $\mathrm{CTAB}$ protocol (Wagner et al., 1987). To characterize Lasiodiplodia spp., the ITS region and elongation factor tef-1 $\alpha$ as phylogenetic markers were used, as recommend in TrunkDiseaseID.org (http://www. grapeipm.org/d.live/) (Lawrence et al., 2017). The oligo- 
nucleotide primers EF1-728F (5'-CATCGAGAAGTTCGAGAAGG-3') and EF1-986R (5'-TACTTGAAGGAACCCTTACC-3') were used to amplify part of the translation elongation factor-1a (tef-1a) gene (Carbone and Kohn, 1999); and ITS1 (5'-TCCGTAGGTGAACCTGCGG-3') and ITS4 (5'-TCCTCCGCTTATTGATATGC-3') were used to amplify the ITS region of the nuclear ribosomal DNA, including the 5.8S gene (White et al., 1990). Each PCR reaction contained $2.5 \mu \mathrm{L}$ of $10 \times$ PCR buffer $(100$ $\mathrm{mM}$ Tris- $\mathrm{HCl}, \mathrm{pH} 8.3$ at $25^{\circ} \mathrm{C} ; 500 \mathrm{mM} \mathrm{KCl} ; 15 \mathrm{mM}$ $\mathrm{MgCl}_{2} ; 0.01 \%$ gelatin), $0.5 \mu \mathrm{L}$ of $20 \mathrm{mM}$ dNTPs, $0.625 \mu \mathrm{L}$ of $10 \mu \mathrm{M}$ of each primer, $0.125 \mu \mathrm{L}$ of Taq DNA polymerase (GoTaq ${ }^{\oplus}$ DNA polymerase, 5 units $\mu \mathrm{L}^{-1}$; Promega), and $1 \mu \mathrm{L}$ of $30 \mathrm{ng} \cdot \mu \mathrm{L}^{-1}$ template DNA, adjusted with purified water to a final volume of $25 \mu \mathrm{L}$. Amplification reactions were carried out in a Bio-Rad T-100 thermal cycler set to the following conditions: for tef- $1 \alpha$, an initial cycle of $95^{\circ} \mathrm{C}$ for $3 \mathrm{~min}$, followed by $35 \mathrm{cycles}$ of $95^{\circ} \mathrm{C}$ for $30 \mathrm{~s}, 55^{\circ} \mathrm{C}$ for $30 \mathrm{~s}$, and $72^{\circ} \mathrm{C}$ for $1 \mathrm{~min}$; for ITS region, an initial cycle of $94^{\circ} \mathrm{C}$ for $2 \mathrm{~min}$, followed by 35 cycles of $94^{\circ} \mathrm{C}$ for $1 \mathrm{~min}$, $58^{\circ} \mathrm{C}$ for $1 \mathrm{~min}$, and $72^{\circ} \mathrm{C}$ for $90 \mathrm{~s}$. Both programmes had a final cycle of $72^{\circ} \mathrm{C}$ for $10 \mathrm{~min}$. Once observed in electrophoresis gels, PCR reactions were purified using the GeneJet PCR purification kit (Thermo Scientific), and purified products were sequenced by Eton Bioscience Inc.

\section{Phylogenetic analyses}

The sequences were analyzed using BioEdit v.7.0.5.3 (Hall, 1999) and a BLASTn analysis was carried out. Sequences with the greatest similarity were downloaded from the GenBank (Table 1) and aligned with ClustalW (pairwise alignment parameters: gap opening 10, gap extension 0.1, and multiple alignment parameters: gap opening 10, gap extension 0.2 . Transition weight was set to 0.5 , and delay divergent sequences to $25 \%$ ) (Thompson et al., 1994). The alignment was adjusted manually where necessary. Alignment of ITS and tef-1a were imported in BioEdit v.7.0.5.3 to obtain the concatenated matrix. Maximum Likelihood (ML) and Maximum Parsimony (MP) analyses were performed using MEGA-X (Kumar et al., 2018), based on the concatenated sequence alignment. The best model of nucleotide substitution was selected according to the Akaike Information Criterion (AIC). The $\mathrm{T} 3+\mathrm{G}+\mathrm{I}$ model was used for the ML analysis (Tamura, 1992). Parameters for Maximum Likelihood were set to Bootstrap method using 1000 replicates. Initial tree(s) for the heuristic search were obtained automatically by applying the Maximum Parsimony method. Gaps were treated as missing data. The tree was visualized in MX: Tree Explorer. New sequences were deposited in the GenBank (https://www.ncbi.nlm.nih.gov/genbank/) (Table 1).
Determination of optimum growth temperature of selected Lasidioplodia isolates

The optimum growth temperature of identified Lasiodiplodia species was determined. Selected isolates of identified species were grown on PDA plates by inoculating each plate with a 3-mm diam. plug of a 2-d-old colony at the edge of the plate. Three replicates of each isolate for each temperature were included, and plates were then incubated at $20,23,25,28,30,37$, or $40^{\circ} \mathrm{C}$. This temperature range was chosen based on previous reports (ÚrbezTorres et al., 2006; Paolinelli-Alfonso et al., 2016), and considering the prevalent summer temperatures of the zone from which the isolates were obtained. The colony radius was measured every $24 \mathrm{~h}$ for $3 \mathrm{~d}$. The optimum growth temperature was determined as the temperature that produced the maximum mycelial growth rate $(\mathrm{mm}$ $\mathrm{d}^{-1}$, which was calculated using the formula:

$G R=\frac{R_{f}-R_{i}}{T_{f}-T_{i}}$

where: $G R=$ Growth rate, $R_{f}=$ Final colony diam. $(\mathrm{mm})$, $R_{i}=$ Initial colony diam. $(\mathrm{mm}), T_{f}=$ Final time (d) when colony measured, and $T_{i}=$ Initial time (day 1 ).

Production of aerial mycelium in Lasiodiplodia spp.

To evaluate aerial mycelium production as a phenotypic characteristic to differentiate among species, $2 \mathrm{~d}$-old cultures of selected isolates were each used to inoculate a $3 \mathrm{~mm}$ diam. plug of each culture into a glass tube containing $5 \mathrm{~mL}$ of PDA medium. Tubes were incubated at $28^{\circ} \mathrm{C}$ for $5 \mathrm{~d}$ and the elevations of mycelia were measured.

\section{Pathogenicity tests of selected Lasiodiplodia isolates}

Based on the analyses of the morphological and genetic results, the isolates MXL28BC, MXCS01BC, MX50BC, MXV5BC, MXVSM1b, MXVSM6, MXVSM16a, MXVSM18, and MXVS21b were selected for pathogenicity tests. Grapevine plants of 'Cabernet Sauvignon' were used to evaluate the pathogenicity of these Lasiodiplodia isolates. Inoculation of each test plant was carried out through a mechanical wound in woody tissue made with a drill bit ( $2 \mathrm{~mm}$ diam.), and a mycelium plug of a selected isolate was placed inside the hole. An isolate of L. gilanensis UCD256Ma (formerly L. theobromae) (Úrbez-Torres et al., 2006; Obrador-Sánchez and Hernandez-Martinez, 2020) was used for comparisons. 
Table 1. List of GenBank and culture accession numbers of Lasiodiplodia spp. used in this study for phylogenetic analyses.

\begin{tabular}{|c|c|c|c|c|c|}
\hline \multirow{2}{*}{ Species } & \multirow{2}{*}{ Isolate } & \multirow{2}{*}{ Host } & \multirow{2}{*}{ Origin } & \multicolumn{2}{|c|}{ GeneBank accession number } \\
\hline & & & & ITS & tef- $1 \alpha$ \\
\hline $\begin{array}{l}\text { Lasiodiplodia } \\
\text { brasiliensis }\end{array}$ & CMM2184 & Carica papaya & Brazil & KC484801 & KC481531 \\
\hline L. brasiliensis & CMM2185 & Carica papaya & Brazil & KC484800 & KC481530 \\
\hline L. brasiliensis & CMM2186 & Carica papaya & Brazil & KC484812 & KC481542 \\
\hline L. brasiliensis & CMM2188 & Carica papaya & Brazil & KC484807 & KC481537 \\
\hline L. brasiliensis & CMM2212 & Carica papaya & Brazil & KC484806 & KC481536 \\
\hline L. brasiliensis & UCD1012BC & Vitis vinifera & USA & EU012372 & EU012392 \\
\hline L. brasiliensis & UCD916SN ${ }^{a}$ & Vitis vinifera & USA & EU012366 & EU012386 \\
\hline L. brasiliensis & UCD923SN ${ }^{\mathrm{a}}$ & Vitis vinifera & USA & EU012371 & EU012391 \\
\hline L. brasiliensis & MXBCL28 & Vitis vinifera & Mexico & MT663281 & MT711988 \\
\hline L. brasiliensis & MXVSCC1 & Vitis vinifera & Mexico & MT663282 & MT711989 \\
\hline L. brasiliensis & MXVS15a & Vitis vinifera & Mexico & MT663283 & MT711990 \\
\hline L. brasiliensis & MXVS16a & Vitis vinifera & Mexico & MT663284 & MT711991 \\
\hline L. brasiliensis & MXVS18 & Vitis vinifera & Mexico & MT663285 & MT711992 \\
\hline L. brasiliensis & MXVS19a & Vitis vinifera & Mexico & MT663302 & MT712009 \\
\hline L. citricola & IRAN1522C & Citrus sp. & Iran & GU945354 & GU945340 \\
\hline L. citricola & IRAN1521C & Citrus sp. & Iran & GU945353 & GU945339 \\
\hline L. crassispora & WAC12533 & Santalum album & Australia & DQ103550 & DQ103557 \\
\hline L. crassispora & CBS110492 & Unknown & Unknown & EF622086 & EF622066 \\
\hline L. crassispora & MXBCV5 & Vitis vinifera & Mexico & MT663286 & MT711993 \\
\hline L. crassispora & MXVS1b & Vitis vinifera & Mexico & MT663287 & MT711994 \\
\hline L. euphorbicola & CMM 4616 & Vitis vinifera & Brazil & MG954348 & MG979518 \\
\hline L. euphorbicola & CMM 4597 & Vitis vinifera & Brazil & MG954347 & MG979517 \\
\hline L. exigua & BL104 & Retama raetam & Tunisia & KJ638317 & KJ638336 \\
\hline L. exigua & BL184 & Retama raetam & Tunisia & KJ638318 & KJ638337 \\
\hline L. exigua & BL185 & Retama raetam & Tunisia & KJ638319 & KJ638338 \\
\hline L. exigua & BL186 & Retama raetam & Tunisia & KJ638320 & KJ638339 \\
\hline L. exigua & BL187 & Retama raetam & Tunisia & KJ638321 & KJ638340 \\
\hline L. exigua & PD161 & Pistacia vera & USA & GU251122 & GU251254 \\
\hline L. exigua & MXBCV4 & Vitis vinifera & Mexico & MT663288 & MT711995 \\
\hline L. exigua & MXBCV6 & Vitis vinifera & Mexico & MT663289 & MT711996 \\
\hline L. exigua & MXBCV7 & Vitis vinifera & Mexico & MT663290 & MT711997 \\
\hline L. exigua & MXVS2Ta & Vitis vinifera & Mexico & MT663291 & MT711998 \\
\hline L. exigua & MXVS5a & Vitis vinifera & Mexico & MT663301 & MT712008 \\
\hline L. exigua & MXVS6a & Vitis vinifera & Mexico & MT663292 & MT711999 \\
\hline L. exigua & MXVS16b & Vitis vinifera & Mexico & MT663293 & MT712000 \\
\hline L. exigua & MXVS20 & Vitis vinifera & Mexico & MT663294 & MT712001 \\
\hline L. exigua & MXVS21a & Vitis vinifera & Mexico & MT663295 & MT712002 \\
\hline L. exigua & MXVS21b & Vitis vinifera & Mexico & MT663296 & MT712003 \\
\hline L. exigua & MXVSS2 & Vitis vinifera & Mexico & MT663303 & MT712010 \\
\hline L. exigua & MXVSSC1 & Vitis vinifera & Mexico & MT663297 & MT712004 \\
\hline L. exigua & MXVSV1 & Vitis vinifera & Mexico & MT663298 & MT712005 \\
\hline L. gilanensis & IRAN1523C & Unknown & Iran & GU945351 & GU945342 \\
\hline L. gilanensis & IRAN1501C & Unknown & Iran & GU945352 & GU945341 \\
\hline L. gilanensis & $\mathrm{UCD} 256 \mathrm{Ma}^{\mathrm{a}}$ & Vitis vinifera & USA & DQ233594 & GU294742 \\
\hline L. gilanensis & MXBC50 & Vitis vinifera & Mexico & MT663299 & MT712006 \\
\hline L. gilanensis & MXBCCS01 & Vitis vinifera & Mexico & MT663300 & MT712007 \\
\hline L. gonubiensis & CMW 14077 & Syzygium cordatum & South Africa & AY639595 & DQ103566 \\
\hline
\end{tabular}


Table 1. (Continued).

\begin{tabular}{|c|c|c|c|c|c|}
\hline \multirow{2}{*}{ Species } & \multirow{2}{*}{ Isolate } & \multirow{2}{*}{ Host } & \multirow{2}{*}{ Origin } & \multicolumn{2}{|c|}{ GeneBank accession number } \\
\hline & & & & ITS & tef- $1 \alpha$ \\
\hline L. gonubiensis & CMW 14078 & Syzygium cordatum & South Africa & AY639594 & DQ103565 \\
\hline L. iraniensis & IRAN1502C & Juglans sp. & Iran & GU945347 & GU945335 \\
\hline L. iraniensis & IRAN921C & Mangifera indica & Iran & GU945346 & GU945334 \\
\hline L. margaritacea & CBS122519 & Adansonia gibbosa & Australia & EU144050 & EU144065 \\
\hline L. margaritacea & CBS122065 & Adansonia gibbosa & Australia & EU144051 & EU144066 \\
\hline L. mediterránea & BL101 & Vitis vinifera & Italy & KJ638311 & KJ638330 \\
\hline L. mediterranea & BL1 & Quercus ilex & Italy & KJ638312 & KJ638331 \\
\hline L. missouriana & UCD2193MO & Vitis sp. & USA & HQ288225 & HQ288267 \\
\hline L. missouriana & UCD2199MO & Vitis sp. & USA & HQ288226 & HQ288268 \\
\hline L. parva & CBS 456.78 & Cassava field-soil & Colombia & EF622083 & EF622063 \\
\hline L. parva & CBS 494.78 & Cassava field-soil & Colombia & EF622084 & EF622064 \\
\hline L. pseudotheobromae & CBS116459 & Gmelina arborea & Costa Rica & EF622077 & EF622057 \\
\hline L. pseudotheobromae & CBS447.62 & Citrus aurantium & Suriname & EF622081 & EF622060 \\
\hline L. pyriformis & CBS 121770 & Acacia mellifera & Nambia & EU101307 & EU101352 \\
\hline L. pyriformis & CBS 121771 & Acacia mellifera & Nambia & EU101308 & EU101353 \\
\hline L. subglobosa & CMM4046 & Jatropha curcas & Brazil & KF234560 & KF226723 \\
\hline L. subglobosa & CMM3872 & Jatropha curcas & Brazil & KF234558 & KF226721 \\
\hline L. theobromae & CBS 164.96 & Fruit along coral reef & Papua New Guinea & AY640255 & AY640258 \\
\hline L. theobromae & CBS111530 & Unknown & Unknown & EF622074 & EF622054 \\
\hline L. venezuelensis & WAC12539 & Acacia mangium & Venezuela & DQ103547 & DQ103568 \\
\hline L. venezuelensis & WAC12540 & Acacia mangium & Venezuela & DQ103548 & DQ103569 \\
\hline Diplodia mutila & CBS 136015 & Populus alba & Portugal & KJ361838 & KJ361830 \\
\hline Diplodia seriata & CBS 112555 & Vitis vinifera & Portugal & AY259094 & AY573220 \\
\hline
\end{tabular}

Isolates from this study are highlighted in bold font.

asolates previously identified as L. theobromae.

Plugs of sterile PDA were used in control plants, and all drill wounds were covered with Parafilm. The grapevine plants were left in greenhouse conditions for 2 months. Samples were then taken to measure the length of the necrotic lesion caused by Lasiodiplodia isolates, and attempts were made to recover the inoculated fungus onto PDA. The experiments in plants were conducted twice. Statistical analyses were carried out using oneway ANOVA followed by post hoc Fisher LSD analyses, with $\alpha<0.05$ for determination of significant differences in virulence between isolates using STATISTICA 8.0.

\section{RESULTS}

Host symptoms, and morphological characteristics of fungal isolates

Botryosphaeria dieback symptoms observed on sampled grapevine plants were mainly dead spurs, cordons, and arms, and shorter shoot internodes. The collected wood exhibited wedge-shaped cankers and necrotic lesions in the vascular bundles.

From necrotic tissue placed in PDA, rapid fungus growth was observed after $2 \mathrm{~d}$. From these colonies, 23 fungal isolates with a similar phenotype were recovered, seven from Baja California and sixteen from Sonora. According to their morphological characteristics, these isolates were identified as Lasiodiplodia. Morphological characteristics included initially white colonies with abundant aerial mycelium, which became smoke-gray and produced pycnidia in PDA as they aged (Figure 2). Pycnidium induction allowed observation of hyaline and pigmented conidia in all the isolates (Figure 3). Inside pycnidia, only hyaline aseptate conidia, with granular contents, were observed, while one-septate pigmented conidia with longitudinal striations were mainly found in cirri (Figure 3). The dimensions (length and width) of 30 conidia per isolate were measured, and minimum, maximum, mean, and standard deviations were calculated (Table 2). Statistically significant differences in conidium dimensions were observed among the four analyzed 


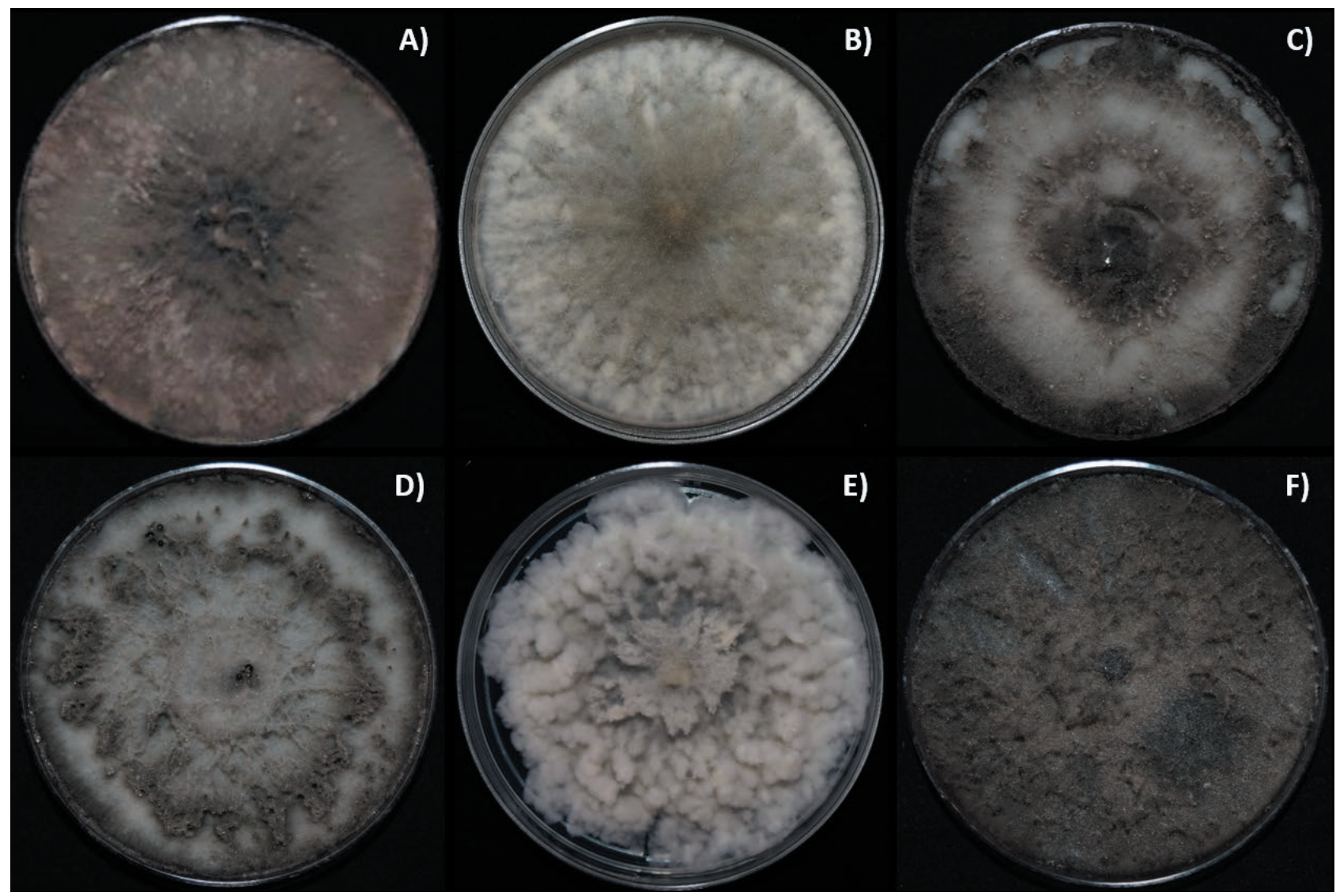

Figure 2. Lasiodiplodia spp. isolates grown on PDA at $30^{\circ} \mathrm{C}$ for 7 d. A) L. brasiliensis MXBCL28, B) L. brasiliensis MXVS18, C) L. exigua MXVS21b, D) L. exigua MXVS5a, E) L. gilanensis MXBCCS01, F) L. crassispora MXVS1b.

Lasiodiplodia species. Isolates characterized as L. gilanensis, MX50 (av. $=28.5 \times 16.6 \mathrm{~mm}$ ), and MXCS01 (av. $=30.2 \times 15.6 \mathrm{~mm}$ ), produced larger and wider conidia than L. brasiliensis, L. crassispora, or L. exigua. Lasiodiplodia brasiliensis and L. crassispora isolates had similar sized conidia (respective mean lengths $=24.0$ and 25.6. $\mathrm{mm}$. The $L$. exigua isolates had shorter conidia (av. = $21.2 \times 12.2 \mathrm{~mm}$ ).

\section{Molecular identification of Lasiodiplodia isolates}

The ITS region and tef-1a loci sequences obtained were, respectively, approx. 500 and 263 bp. The combined dataset comprised 832 characters including gaps after alignment (541 corresponded to the ITS gene and 291 corresponded to the tef 1 gene), and 72 taxa. Diplodia mutila (CBS 136015) and Diplodia seriata (CBS 112555) were used as the outgroup taxa. Maximum parsimony analysis yielded one most parsimonious tree $[($ length $=151, \mathrm{CI}=0.711864$ (0.677885), $\mathrm{RI}=0.922197$,
$\mathrm{RC}=0.714550(0.656479)]$ for all sites and parsimonyinformative sites. Maximum likelihood analysis using the Tamura 3-parameter model resulted in a tree with the $\log$ likelihood value of -2252.61 . The rate variation model allowed for some sites to be evolutionarily invariable $([+I], 41.41 \%$ sites). Estimated base frequencies were: $\mathrm{A}=0.21487, \mathrm{C}=0.28764, \mathrm{G}=0.25966$, and $\mathrm{T}=0.23783$; and a discrete Gamma distribution was used to model evolutionary rate differences among sites [five categories $(+\mathrm{G}$, parameter $=0.5665)]$.

The phylogenetic analysis of the ITS region and tef- $1 \alpha$ revealed that the isolates were of four different Lasiodiplodia spp. (Figure 4). Most of the isolates were L. exigua (syn. Lasiodiplodia mahajangana) (isolates MXBCV4, MXBCV7, MXBCV6, MXVSV1, MXVS5a, MXVSSC1, MXVSS2, MXVS2Ta, MXVS6a, MXVS16b, MXVS20, MXVS21a, and MXVS21b). Six isolates were L. brasiliensis (isolates MXBCL28, MXVSCC1, MXVS15a, MXVS16a, MXVS18, and MXVS19a); two isolates were L. gilanensis (syn. Lasiodiplodia missouriana) (isolates MXBCCS01 and MXBC50); and two isolates were 


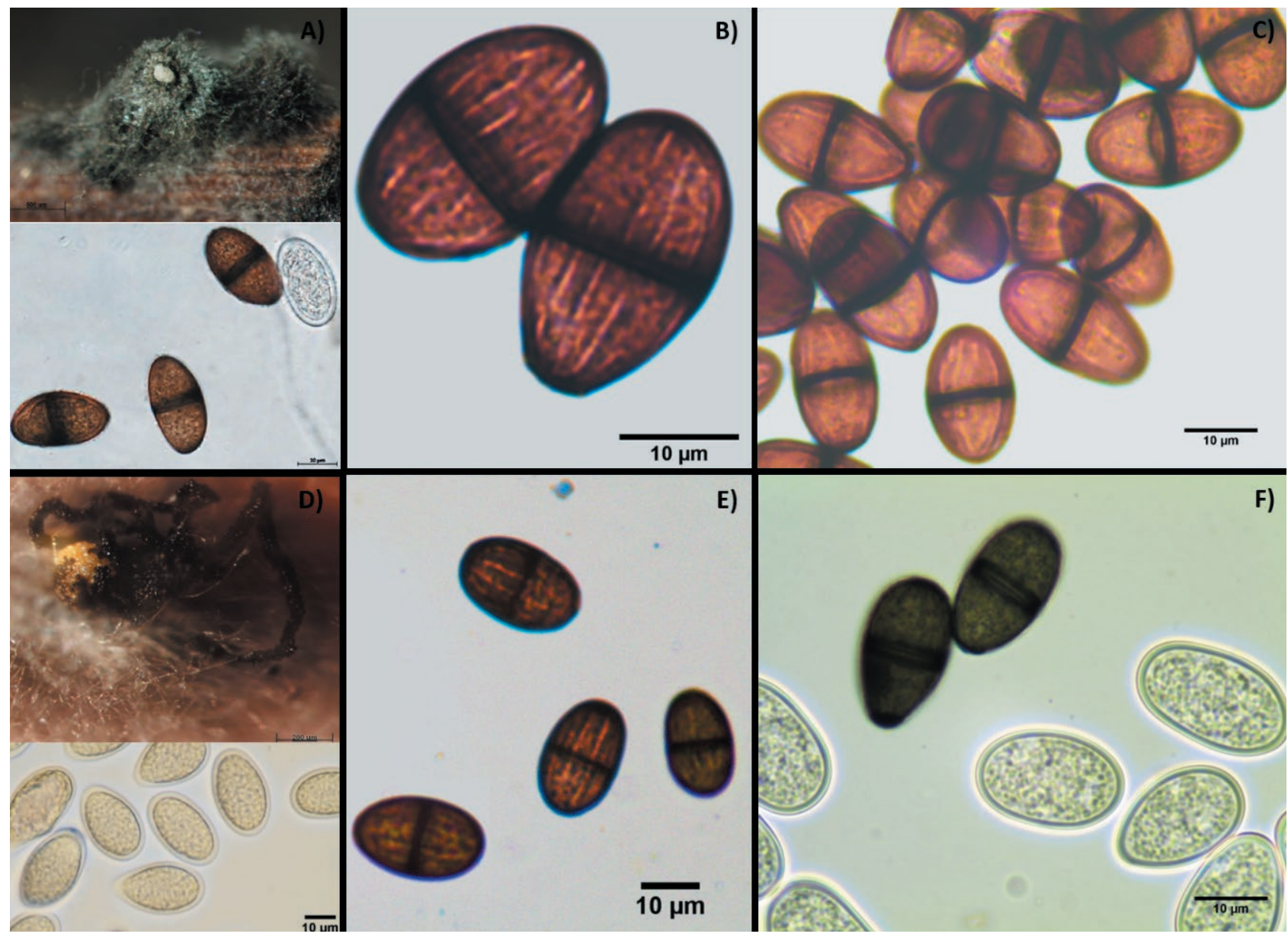

Figure 3. Conidia of Lasiodiplodia spp. isolates. A) L. brasiliensis MXBCL28, B) L. brasiliensis MXVS18, C) L. exigua MXVS21b, D) L. gilanensis MXBCCS01, E) L. crassispora MXVS1b, F) L. exigua MXVS5a.

L. crassispora (syn. Lasiodiplodia pyriformis) (isolates MXBCV5 and MXVS1b). Previously, only L. theobromae had been described in Baja California and Sonora (Úrbez-Torres et al., 2008). Nonetheless, the three $L$. theobromae sensu stricto isolates used as references were clustered separately, and the isolates from the 2008 study of Baja California and Sonora were clustered within the clade of L. brasiliensis (Figure 4, Figure S1).

Optimum growth temperature and aerial mycelium production of Lasiodiplodia spp.

The Lasiodiplodia isolates selected had optimum growth temperatures of $28^{\circ} \mathrm{C}$. Most of the isolates grew at greater than $20 \mathrm{~mm} \mathrm{~d}^{-1}$ at $30^{\circ} \mathrm{C}$ (Table 3). Lasiodiplodia exigua grew at up to a mean of $24.6 \mathrm{~mm} \mathrm{~d}^{-1}$ at $37^{\circ} \mathrm{C}$, and this was the only species that grew at $40^{\circ} \mathrm{C}$. Lasiodiplodia gilanensis had the least mycelium growth rate, with a maximum mean growth rate of $19.8 \mathrm{~mm} \mathrm{~d}^{-1}$ at $28^{\circ} \mathrm{C}$.
All the Lasiodiplodia isolates produced aerial mycelium, but in L. gilanensis this was less (mean = $0.8 \pm 0.4 \mathrm{~mm}$ ) than for the other species. The most abundant and longest aerial mycelium was observed in L. exigua isolate MXVS5a $(16 \pm 4.8 \mathrm{~mm})$, followed by L. brasiliensis $(9.0 \pm 2.56 \mathrm{~mm})$. The species Lasiodiplodia crassispora produced less abundant aerial mycelium $(5.4 \pm 2.3 \mathrm{~mm})$ than the other species, and this species melanized more rapidly than the other species (Figure 5).

Evaluation of the pathogenicity of selected isolates of Lasiodiplodia spp.

Pathogenicity assays on grapevine plants showed that two-months post inoculation L. brasiliensis MXBCL28 and MXVS18, and L. gilanensis MXCS01 were the most virulent isolates (Figure 5, C, D, and F), in the woody shoots induced necrotic lesions up to $6 \mathrm{~cm}$ in 
Table 2. Conidium dimensions of the Lasiodiplodia spp. isolates from this study.

\begin{tabular}{|c|c|c|c|}
\hline Isolate & Origin & Conidium size $^{\mathrm{a}}$ & Mean $\pm \mathrm{SD}^{\mathrm{b}}$ \\
\hline \multicolumn{4}{|c|}{ Lasiodiplodia brasiliensis $^{\mathrm{b}}$} \\
\hline MXBCL28 & Valle de Guadalupe, B.C. & $(21.9-) 24-28.4 \times(12.8-) 13.6-14.7$ & $24.3 \pm 1.4 \times 13.7 \pm 0.7$ \\
\hline MXVSCC1 & Hermosillo, Sonora & $(20.4-) 24.6-27.1 \times(11.3-) 12.5-14.8$ & $23.7 \pm 1.7 \times 12.8 \pm 0.8$ \\
\hline MXVS15a & Hermosillo, Sonora & $(20.3-) 22.3-24.6 \times(11.5-) 12.5-14.4$ & $22.8 \pm 1 \times 12.5 \pm 0.7$ \\
\hline MXVS16a & Hermosillo, Sonora & $(22.1-) 26.8-27.6 \times(10.6-) 11.7-13.1$ & $24.7 \pm 1.6 \times 11.9 \pm 0.5$ \\
\hline MXVS18 & Hermosillo, Sonora & $(21.3-) 24.8-29.4 \times(11.3-) 13.5-15.2$ & $24.7 \pm 2 \times 13.3 \pm 0.8$ \\
\hline MXVS19a & Hermosillo, Sonora & $(20.1-) 23.3-26.4 \times(11.4) 13.4-16.8$ & $23.2 \pm 1.7 \times 13.3 \pm 1.3$ \\
\hline \multicolumn{4}{|c|}{ 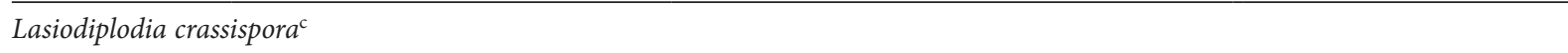 } \\
\hline $\begin{array}{l}\text { MXBCV5 } \\
\text { MXVS1b }\end{array}$ & $\begin{array}{l}\text { Valle de Guadalupe, B.C. } \\
\text { Hermosillo, Sonora }\end{array}$ & $\begin{array}{c}(23.0-) 24.4-29.9 \times(13.3-) 16.7-20.2 \\
(23.7-) 24.6-27.1 \times(13-) 14.7-16.7\end{array}$ & $\begin{array}{l}26.1 \pm 2.2 \times 17.5 \pm 1.7 \\
25.0 \pm 0.9 \times 14.7 \pm 1.1\end{array}$ \\
\hline \multicolumn{4}{|c|}{ Lasiodiplodia exigua ${ }^{\mathrm{a}}$} \\
\hline MXBCV4 & Valle de Guadalupe, B.C. & $(18.6-) 21.1-24.8 \times(11-) 12-13.9$ & $21.5 \pm 1.6 \times 12.2 \pm 0.8$ \\
\hline MXBCV6 & Valle de Guadalupe, B.C. & $(18.4-) 19.2-22.5 \times(10.5-) 11.4-12.7$ & $20.2 \pm 1.1 \times 11.2 \pm 0.7$ \\
\hline MXBCV7 & Valle de Guadalupe, B.C. & $(19.1-) 20.1-21.7 \times(12.0-) 12.9-14.2$ & $20.3 \pm 0.7 \times 12.9 \pm 0.5$ \\
\hline MXVS5a & Hermosillo, Sonora & $(21.1-) 22.5-25.6 \times(11.7-) 13.2-16$ & $22.7 \pm 1.1 \times 13.9 \pm 1.0$ \\
\hline MXVS6a & Hermosillo, Sonora & $(21.0-) 23.4-24.6 \times(11.9-) 12.9-13.9$ & $22.8 \pm 1.0 \times 13 \pm 0.5$ \\
\hline MXVS2Ta & Hermosillo, Sonora & $(19.7-) 21.3-22.8 \times(11.3-) 12.3-12.9$ & $21.3 \pm 0.9 \times 12.2 \pm 0.5$ \\
\hline MXVS16b & Hermosillo, Sonora & $(19.6-) 23-26.9 \times(11.1) 13-14.9$ & $22.5 \pm 2.0 \times 12.9 \pm 0.9$ \\
\hline MXVS20 & Hermosillo, Sonora & $(20.2-) 21.9-23.7 \times(11.2-) 12.7-13.9$ & $22.2 \pm 0.9 \times 12.8 \pm 0.7$ \\
\hline $\begin{array}{l}\text { MXVS21a } \\
\text { MXVS21b }\end{array}$ & $\begin{array}{l}\text { Hermosillo, Sonora } \\
\text { Hermosillo, Sonora }\end{array}$ & $\begin{array}{l}(18.4-) 19.6-23.8 \times(10.1-) 12.5-13.9 \\
(19.3-) 20.3-23.2 \times(10.7-) 11.9-13.4\end{array}$ & $\begin{aligned} 20.6 \pm 1.5 & \times 12.5 \pm 0.9 \\
21 \pm 1.0 & \times 12 \pm 0.7\end{aligned}$ \\
\hline MXVSV1 & Hermosillo, Sonora & $(19.1-) 20.8-23.4 \times(10.2) 12-12.8$ & $20.6 \pm 1.0 \times 11.6 \pm 0.7$ \\
\hline MXVSSC1 & Hermosillo, Sonora & $(18.2-) 19.8-24.1 \times(10.5-) 11.5-13.5$ & $20.8 \pm 1.9 \times 11.7 \pm 0.6$ \\
\hline MXVSS2 & Hermosillo, Sonora & $(18.3-) 20-23 \times(11.4-) 11.9-14.2$ & $20.5 \pm 1.2 \times 12.5 \pm 0.7$ \\
\hline \multicolumn{4}{|c|}{ Lasiodiplodia gilanensis $^{d}$} \\
\hline MXBC50 & Valle de Guadalupe, B.C. & $(25.6-) 28-33.8 \times(15-) 17.1-18.1$ & $28.5 \pm 1.7 \times 16.6 \pm 0.6$ \\
\hline MXNCCS01 & Valle de Guadalupe, B.C. & $(25.4-) 28.9-33 \times(13.8-) 15.4-18.7$ & $30.2 \pm 1.8 \times 15.6 \pm 1.2$ \\
\hline
\end{tabular}

a Minimum size, most repetitive value and maximum size for length and width of 30 conidia selected.

b $\mathrm{SD}=$ standard deviation.

a,b,c,d Means accompanied by the same letters are not significantly different $(\alpha<0.05)$.

length around the inoculation site, and were significantly different from the other inoculated isolates. L. exigua MXVS21b caused necrotic lesions in length, similar to L. gilanensis UCD256Ma (Figure 5 and 6). L. crassispora MXBCV5 and MXVS1b caused lesion below $1 \mathrm{~cm}$ in length (Figure 5 and 6 ) and showed a non-significant difference in comparison to control plants. All isolates were recovered from the inoculate site at three days after incubation at $30^{\circ} \mathrm{C}$ on PDA plates, which confirmed Koch's postulates. Non-necrotic lesions were observed in the control plants, only the wound effect; instead, green tissue was found, which indicated tissue regeneration of the caused wound.

\section{DISCUSSION}

In this study, four Lasiodiplodia species causing Botryosphaeria dieback symptoms were identified from Mexican vineyards. Lasiodiplodia theobromae, the type species of Lasiodiplodia, is one of the most common species associated with Botryosphaeria dieback in grapevine (Úrbez-Torres, 2011; Fontaine et al., 2016), and for several years, it was the only known species within the genus. Later, L. theobromae was shown to be a complex of cryptic species (Alves et al., 2008), which led to taxonomic revision of Lasiodiplodia. As a result, fungal isolates previously reported as $L$. theobromae have been re- 


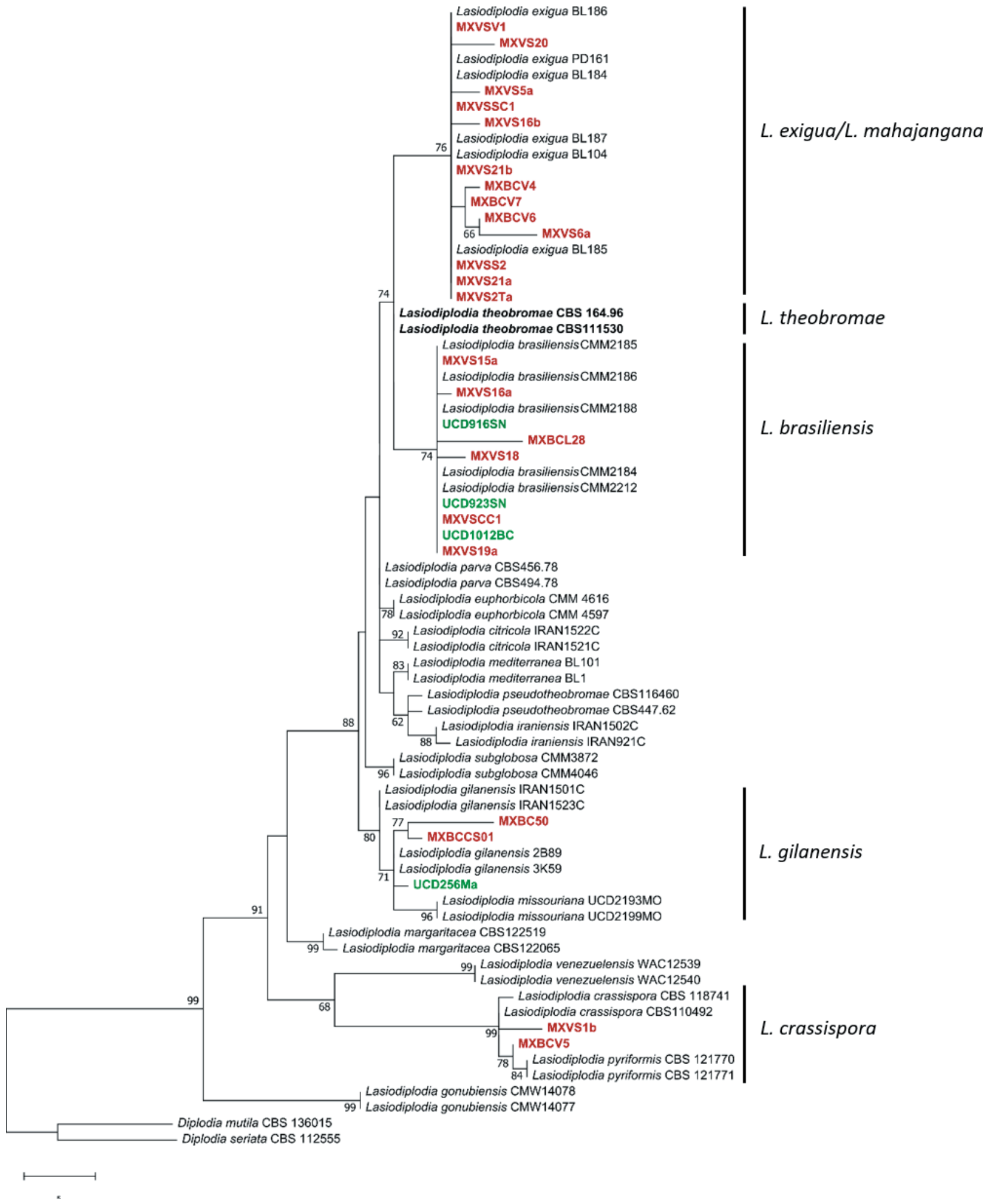

Figure 4. Phylogenetic analysis. Most-parsimonious tree (length $=151)$ obtained from analysis of ITS and tef1 concatenated datasets. Bootstrap values from 1000 replicates greater than 50 are indicated at the nodes. The tree is rooted with Diplodia mutila (CBS 136015) and Diplodia seriata (CBS 112555). The isolates from the present study are indicated in bold red font, isolates previously identified as L. theobromae are indicated in bold green font, and the L. theobromae sensu stricto isolates are indicated in bold black font. 
Table 3. Mean colony diameters at different temperatures for Mexican Lasiodiplodia isolates grown in PDA cultures.

\begin{tabular}{|c|c|c|c|c|c|c|c|}
\hline \multirow{2}{*}{ Isolate } & \multicolumn{7}{|c|}{ Temperature } \\
\hline & $20^{\circ} \mathrm{C}$ & $23^{\circ} \mathrm{C}$ & $25^{\circ} \mathrm{C}$ & $28^{\circ} \mathrm{C}$ & $30^{\circ} \mathrm{C}$ & $37^{\circ} \mathrm{C}$ & $40^{\circ} \mathrm{C}$ \\
\hline \multicolumn{8}{|c|}{ Lasiodiplodia brasiliensis } \\
\hline MXBCL28 & $19.1 \pm 0.7$ & $21.6 \pm 2.4$ & $20 \pm 1.3$ & $28.1 \pm 0.2$ & $20.6 \pm 3.6$ & $6.8 \pm 0.57$ & 0 \\
\hline MXVS18 & $15 \pm 0$ & $20 \pm 0.8$ & $23.1 \pm 1.0$ & $27.3 \pm 1.7$ & $22.0 \pm 1.0$ & $20.0 \pm 1.8$ & 0 \\
\hline \multicolumn{8}{|c|}{ Lasiodiplodia crassispora } \\
\hline MXBCV5 & $12.6 \pm 0.2$ & $17.3 \pm 0.2$ & $19.1 \pm 1.5$ & $23.1 \pm 0.2$ & $20.1 \pm 1$ & $3.8 \pm 0.7$ & 0 \\
\hline \multicolumn{8}{|c|}{ Lasiodiplodia exigua } \\
\hline MXVS5a & $15 \pm 1.3$ & $21.3 \pm 2$ & $19.8 \pm 0.7$ & $28.1 \pm 1.5$ & $20.5 \pm 2.2$ & $21.6 \pm 1$ & $0.5 \pm 0$ \\
\hline MXVS21b & $17.16 \pm 0.2$ & $19.6 \pm 0.5$ & $20.6 \pm 1.5$ & $23 \pm 2.1$ & $22.3 \pm 0.7$ & $24.6 \pm 0.7$ & $0.5 \pm 0$ \\
\hline \multicolumn{8}{|c|}{ Lasiodiplodia gilanensis } \\
\hline MXBC50 & $11 \pm 2.4$ & $8.1 \pm 0.7$ & $5.6 \pm 1.6$ & $6.1 \pm 1.2$ & $11.3 \pm 7.2$ & $5.8 \pm 1.6$ & 0 \\
\hline MXBCCS01 & $16.3 \pm 0.35$ & $17.1 \pm 2.46$ & $17.5 \pm 3.6$ & $19.8 \pm 5.0$ & $18.1 \pm 1.89$ & $9.5 \pm 0.5$ & 0 \\
\hline
\end{tabular}

L. brasiliense

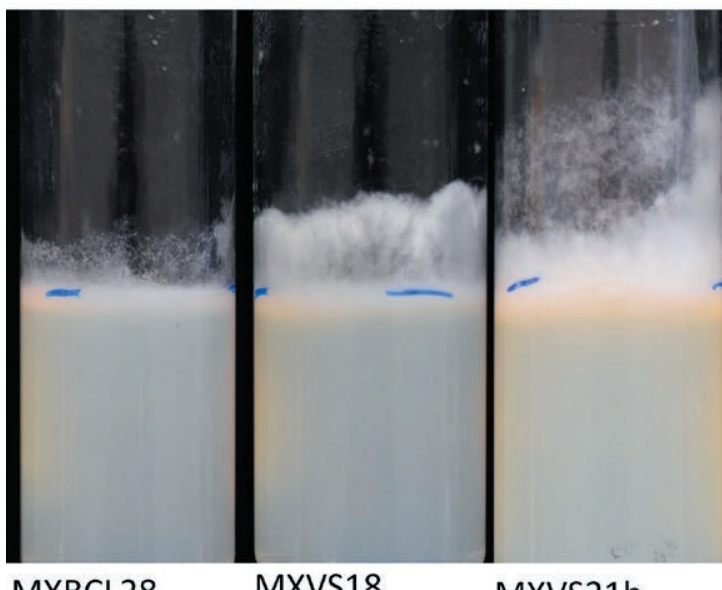

MXBCL28
L. exigua

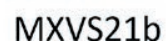

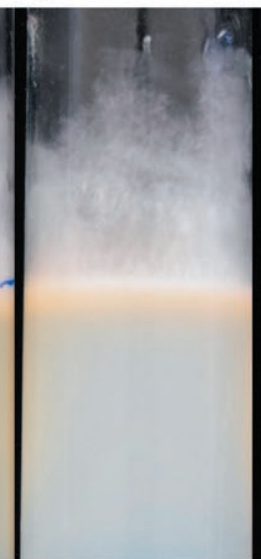

MXVS5a
L. gilanensis

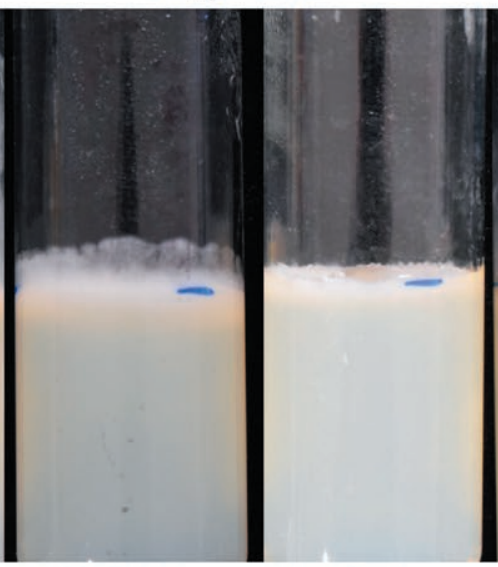

MXBCCS01
L. crassispora

Figure 5. Aerial mycelium growth of different Lasiodiplodia spp. isolated from grapevines in Mexico. The isolates were grown in glass tubes containing PDA medium for $5 \mathrm{~d}$ at $28^{\circ} \mathrm{C}$.

classified as new species (Dissanayake et al., 2016; Cruywagen et al., 2017; Mehl et al., 2017; Tibpromma et al., 2018). Some species were subsequently reduced to synonymy (Zhang et al., 2021). The fungal rDNA internal transcribed spacer region (ITS) is the primary barcode used to identify fungal species, but in Lasiodiplodia spp., this region has low interspecific variation. The translation elongation factor $1-\alpha(t e f-1 \alpha)$ is more variable than ITS, and has been recommended as a secondary barcode region to estimate species identity for Botryosphaeriaceae (Lawrence et al., 2017), and this locus allowed us to segregate $L$. brasiliensis from $L$. theobromae.

Pathogens associated with wood dieback diseases are generally found in vineyards that are at least 10 years old (Gubler et al., 2005), but we have isolated these fungi in younger vineyards in Mexico. Lasiodiplodia exigua, L. brasiliensis, and L. crassispora were recovered from the two Mexican viticulture areas (Baja California and Sonora), whereas L. gilanensis was only found in Baja California. Lasiodiplodia exigua was the most prevalent species. Previously, only $L$. theobromae was reported in Mexico in grapevine (Úrbez-Torres et al., 2008), but our phylogenetic analyses indicated that those isolates clustered with $L$. brasiliensis, suggesting that $L$. brasiliensis has been in Mexico for a long time.

Production of reddish-pink pigment by the isolates of L. brasiliensis and L. gilanensis was observed. This characteristic has been reported in other species 


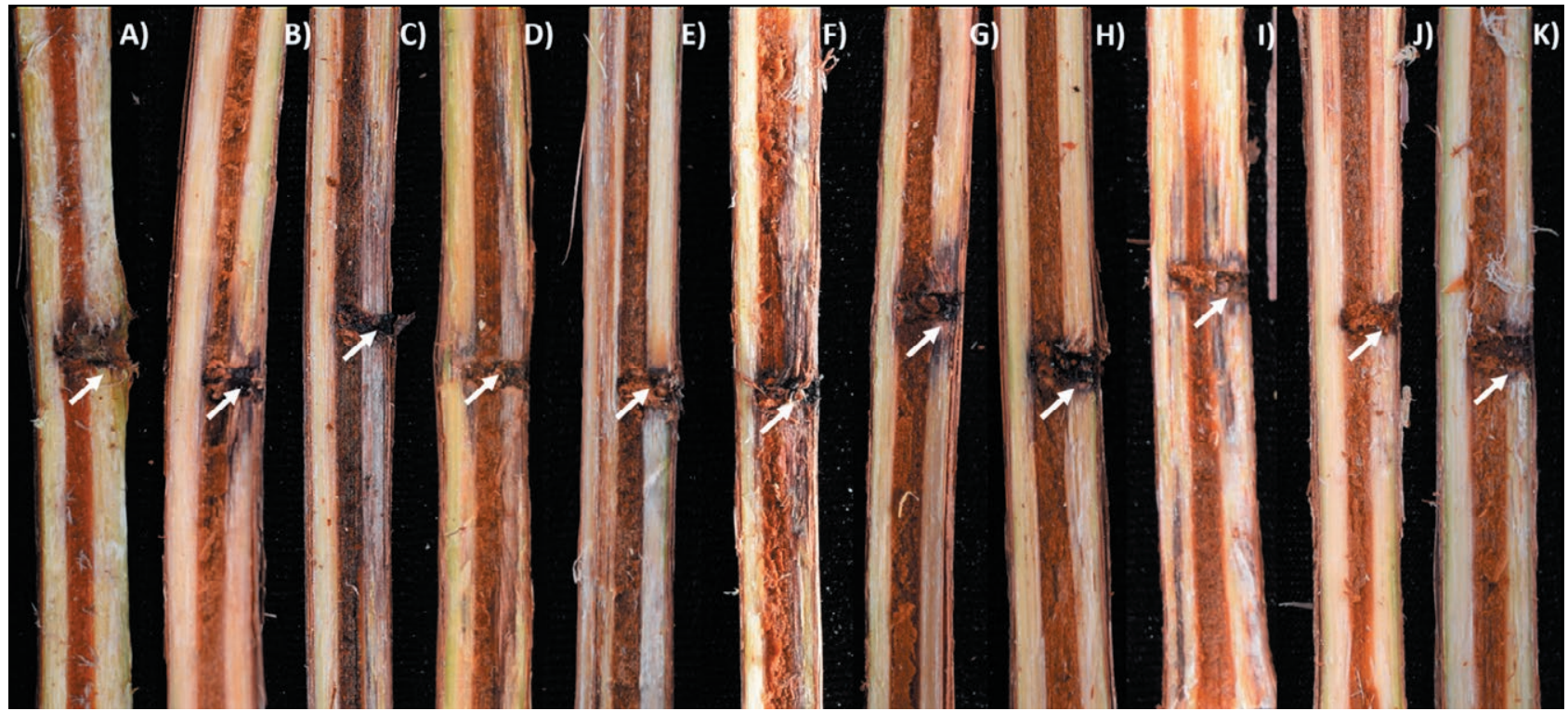

Figure 6. Grapevine woody shoots showing dark-brown lesions at 2-months post inoculation with Lasiodiplodia isolates. A) Control plant (PDA), B) L. gilanensis UCD256Ma, C) L. brasiliensis MXBCL28, D) L. brasiliensis MXVS18, E) L. brasiliensis MXVS16a F) L. gilanensis MXBCCS01, G) L. gilanensis MXBC50, H) L. exigua MXVS6a, I) L. exigua MXVS21b J) L. crassispora MXVS1b, and K) L. crassispora MXBCV5. White arrows indicate the point of inoculation.

including L. pseudotheobromae, L. parva, and L. theobromae (Alves et al., 2008; Abdollahzadeh et al., 2010). Although L. missouriana has been reduced to synonymy with L. gilanensis (Zhang et al., 2021), conidium dimensions of the Mexican isolates of L. gilanensis (isolates MX50 and MXSC01) and one from California, USA (isolate UCD256Ma) were larger (av. $=29.6 \times 15.6$ $\mu \mathrm{m}$ ) than those for L. missouriana (av. $=18.5 \times 9.8 \mu \mathrm{m}$ ) from Missouri, USA (Phillips et al., 2013). On the other hand, $L$. theobromae (av. $= \pm \mathrm{SD}=26.2 \pm 2.6 \times 14.2$ $\pm 1.2 \mu \mathrm{m}$ ) (Phillips et al., 2013) had conidium dimensions similar to those for $L$. brasiliensis (av. $\pm \mathrm{SD}=$ $26.01 \pm 1.36 \times 14.64 \pm 1.16 \mu \mathrm{m}$ ) (Netto et al., 2014), making these species difficult to distinguish based solely on morphological traits. In the present study, aerial mycelium height was another morphological characteristic evaluated, and the observed differences suggested that this trait could help with the differentiation of Lasiodiplodia species.

The pathogenicity tests showed that the L. brasiliensis isolates MXBCL28 and MXVS18, and L. gilanensis isolate MXCS01 were the most virulent to grapevine plants 'Cabernet Sauvignon'. These isolates caused necrotic lesions to the host vascular systems at 2 months post-inoculation. Lasiodiplodia brasiliensis was also reported for the first time on grapevine in Brazil, and this was the most virulent species on green shoots, followed by $L$. theobromae (Correia et al., 2016). Lasidiplo-

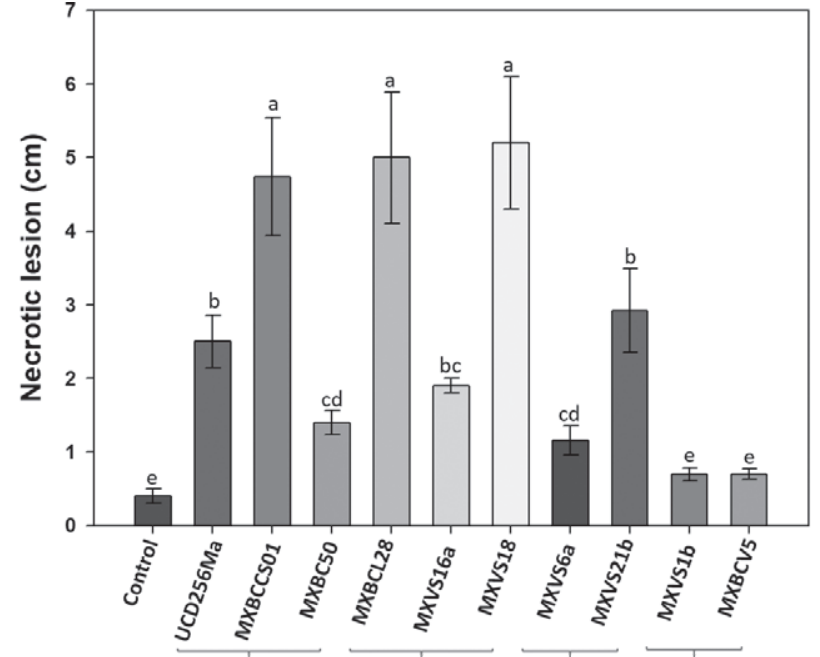

Figure 7. Mean lesion length caused by Lasiodiplodia isolates in grapevine plants 2-months post inoculation under greenhouse conditions. Bars indicate the standard deviation of each treatment. Significance letters were grouped based on Fisher's analysis $(\mathrm{P}<0.05)$; Bars indicate standard deviations. Means accompanied by the same letters are not significantly different $(\alpha<0.05)$

dia gilanensis was described for the first time from Iran, from an unknown tree showing branch dieback, cankers, and fruit rot (Abdollahzadeh et al., 2010). Considering isolate UCD256Ma, formerly identified as L. theo- 
bromae (Úrbez-Torres et al., 2006) belongs to L. gilanensis, the present study data supports taxonomic reassignment. Lasiodiplodia missouriana has been reduced to synonymy with L. gilanensis (Zhang et al., 2021). Lasiodiplodia missouriana was isolated from grapevines in 2011, and was one of the most aggressive species to grapevine (Úrbez-Torres et al., 2012), confirming results from the present study.

Lasiodiplodia exigua isolates MXVS6a and MXVS21b were of different virulence than $L$. brasiliensis and L. gilanensis isolates. Lasiodiplodia exigua was first isolated from broom bush (Retama raetam) in Tunisia (Linaldeddu et al., 2015), and was reported to cause brown discolouration and streaks in grapevine wood (Akgül et al., 2019). The L. crassispora isolates MXBCV5 and MXVS1b from the present study were the least virulent, which is similar to the results from previous studies (Correia et al., 2016).

Grapevine plants are susceptible to several different wood pathogens during the pruning period, so it is important to consider factors such as climatic conditions and life cycles of GTDs pathogens (Rolshausen et al., 2010; Agustí-Brisach et al., 2015; Gramaje et al., 2018; Waite et al., 2018). Spread of fungus pathogens involved in Botryosphaeria dieback within vineyards is linked with rainfall and associated wind dispersal of inocula (Mehl et al., 2017). Lasiodiplodia has been reported to be prevalent in regions with high temperatures and low precipitation (Úrbez-Torres, 2011; Gispert et al., 2020). The isolates examined in the present study had optimum growth temperatures of $28^{\circ} \mathrm{C}$, but all grew at $37^{\circ} \mathrm{C}$, and the isolates of L. exigua grew at $40^{\circ} \mathrm{C}$. This could be an adaptation of $L$. exigua to extreme hot weather conditions. This species is the most commonly found in the Baja California and Sonora grape-growing regions. Even when the other isolates did not grow at $40{ }^{\circ} \mathrm{C}$, they recovered their average growth once they were transferred to room temperature, except for L. gilanensis isolate MXBC50. These fungi probably entered a dormant state that recovers when temperatures decrease. This could explain why L. gilanensis is the most common species in Baja California and Sonora, where prevalent climate conditions are annual precipitation of $280 \mathrm{~mm}$ and temperatures greater than $40^{\circ} \mathrm{C}$ during the summer, conditions which favour growth of L. gilanensis. More studies are required of these fungi under extreme growing conditions. However, the present study has contributed to recognizing GTD pathogen species present in Mexico's most economically important viticulture region, representing the first step for epidemiological studies to assist controlling the spread of these pathogens.

\section{ACKNOWLEDGMENTS}

Edelweiss A. Rangel-Montoya received a scholarship from CONACYT. Marcos Paolinelli acknowledges the support provided by a CONICET postdoctoral fellowship.

\section{LITERATURE CITED}

Abdollahzadeh J., Javadi A., Mohammadi Goltapeh E., Zare R., Phillips A.J., 2010. Phylogeny and morphology of four new species of Lasiodiplodia from Iran. Persoonia: Molecular Phylogeny and Evolution of Fungi 25: 1-10.

Agustí-Brisach C., León M., García-Jiménez J., Armengol J., 2015. Detection of grapevine fungal trunk pathogens on pruning shears and evaluation of their potential for spread of infection. Plant Disease 99: 976-981.

Akgül D.S., Savaş N.G., Özarslandan M., 2019. First report of wood canker caused by Lasiodiplodia exigua and Neoscytalidium novaehollandiae on grapevine in Turkey. Plant Disease 103: 1036.

Alves A., Crous P.W., Correia A., Phillips A.J.L., Alves A., 2008. Morphological and molecular data reveal cryptic speciation in Lasiodiplodia theobromae. Fungal Diversity 28: 1-13.

Bertsch C., Ramírez-Suero M., Magnin-Robert M., Larignon P., Chong J., ... Fontaine F., 2013. Grapevine trunk diseases: complex and still poorly understood. Plant Pathology 62: 243-265.

Billones-Baaijens R., Savocchia S., 2019. A review of Botryosphaeriaceae species associated with grapevine trunk diseases in Australia and New Zealand. Australasian Plant Pathology 48: 3-18.

Carbone I., Kohn L., 1999. A method for designing primer sets for speciation studies in filamentous Ascomycetes. Mycologia 91: 553-556.

Correia K.C., Silva M.A., de Morais M.A., Jr. Armengol J., Phillips A.J.L., ... Michereff S.J., 2016. Phylogeny, distribution and pathogenicity of Lasiodiplodia species associated with dieback of table grape in the main Brazilian exporting region. Plant Pathology 65: 92-103.

Cruywagen E.M., Slippers B., Roux J., Wingfield M.J., 2017. Phylogenetic species recognition and hybridisation in Lasiodiplodia: a case study on species from baobabs. Fungal Biology 121: 420-436.

Dissanayake A.J., Phillips A.J.L., Li X.H., Hyde K.D., 2016. Botryosphaeriaceae: current status of genera and species. Mycosphere 7: 1001-1073. 
Fontaine F., Pinto C., Vallet J., Clément C., Gomes A.C., Spagnolo A., 2016. The effects of grapevine trunk diseases (GTDs) on vine physiology. European Journal of Plant Pathology 144: 707-721.

García-Robles J.M., Tobón-Quijano J.I., Bringas-Taddei E., Mercado-Ruiz J.N., Luchsinger-Lagos L., BáezSañudo R., 2007. Daños y desórdenes fisiológicos en uva de mesa sonorense después del preenfriado y almacenamiento. Revista Iberoamericana de Tecnología Postcosecha 8: 89-100.

Gispert C., Kaplan J.D., Deyett E., Rolshausen P.E., 2020. Long-Term Benefits of Protecting table grape vineyards against trunk diseases in the California desert. Agronomy 10: 1895.

González-Andrade S., 2015. Cadena de valor económico del vino de Baja California, Mexico. Estududios fronterizos 16: 163-193.

Gramaje D., Armengol J., 2011. Fungal trunk pathogens in the grapevine propagation process potencial inoculum sources, detection, identification, and management strategies. Plant Disease 95: 1040-1055.

Gramaje D., Úrbez-Torres J.R., Sosnowski M.R., 2018. Managing grapevine trunk diseases with respect to etiology and epidemiology: current strategies and future prospects. Plant Disease 102: 12-39.

Gubler W.D., Rolshausen P.E., Trouillase F.P., Úrbez J.R., Voegel T., 2005. Grapevine trunk diseases in California. Practical Winery \& Vineyard Jan/Feb: 6-25.

Hall T.A., 1999. BioEdit: a user-friendly biological sequence alignment editor and analysis program for Windows 95/98/NT. Nucleic acids symposium series 41: 95-98.

Kumar S., Stecher G., Li M., Knyaz C., and Tamura K., 2018. MEGA X: Molecular Evolutionary Genetics Analysis across computing platforms. Molecular Biology and Evolution 35: 1547-1549.

Lawrence D.P., Travadon R., Nita M., Baumgartner K., 2017. TrunkDiseaseID. org: A molecular database for fast and accurate identification of fungi commonly isolated from grapevine wood. Crop Protection 102: $110-117$.

Linaldeddu B.T., Deidda A., Scanu B., Franceschini A., Serra S., ... Phillips A.J.L., 2015. Diversity of Botryosphaeriaceae species associated with grapevine and other woody hosts in Italy, Algeria and Tunisia, with descriptions of Lasiodiplodia exigua and Lasiodiplodia mediterranea sp. nov. Fungal Diversity 71: 201214.

Mehl J., Wingfield M.J., Roux J., Slippers B., 2017. Invasive everywhere? Phylogeographic analysis of the globally distributed tree pathogen Lasiodiplodia theobromae. Forests 8: 1-22.
Netto M.S., Assunção I.P., Lima G.S., Marques M.W., Lima W.G., ... Câmara M.P., 2014. Species of Lasiodiplodia associated with papaya stem-end rot in Brazil. Fungal Diversity 67: 127-141.

Obrador-Sánchez J.A., Hernandez-Martinez R., 2020. Microscope observations of Botryosphaeriaceae spp. in the presence of grapevine wood Phytopathologia Mediterranea 59: 119-129.

Paolinelli-Alfonso M., Villalobos-Escobedo J.M., Rolshausen P., Herrera-Estrella A., Galindo-Sánchez C., ... Hernandez-Martinez R., 2016. Global transcriptional analysis suggests Lasiodiplodia theobromae pathogenicity factors involved in modulation of grapevine defensive response. BMC Genomics 17,615 .

Phillips A.J.L., Alves A., Abdollahzadeh J., Slippers B., Wingfield, M.J., ... Crous P.W., 2013. The Botryosphaeriaceae: genera and species known from culture. Studies in Mycology 76: 51-167.

Punithalingam E., 1976. Botryodiplodia theobromae. IMI Descriptions of Fungi and Bacteria 519: 1-2.

Rolshausen P.E., Úrbez-Torres J.R., Rooney-Latham S., Eskalen A., Smith R.J., Gubler W.D., 2010. Evaluation of pruning wound susceptibility and protection against fungi associated with grapevine trunk diseases. American Journal of Enology and Viticulture 61: 113-119.

Rolshausen P.E., Akgül D.S., Perez R., Eskalen A., Gispert C., 2013. First report of wood canker caused by Neoscytalidium dimidiatum on grapevine in California. Plant Disease 97: 1511-1511.

SIAP Servicio de Información y Estadística Agroalimentaria y Pesquera, 2019. Ministerio de Agricultura de Mexico, Secretaría de Agricultura, Ganadería, Desarrollo Rural, Pesca y Alimentación (SAGARPA).

Slippers B., Smit W.A., Crous P.W., Coutinho T.A., Wingfield B.D., Wingfield M.J., 2007. Taxonomy, phylogeny and identification of Botryosphaeriaceae associated with pome and stone fruit trees in South Africa and other regions of the world. Plant Pathology 56: 128-139.

Stempien E., Goddard M.L., Wilhelm K., Tarnus C., Bertsch C., Chong, J., 2017. Grapevine Botryosphaeria dieback fungi have specific aggressiveness factor repertory involved in wood decay and stilbene metabolization. PloS one 12: e0188766.

Tamura K., 1992. Estimation of the number of nucleotide substitutions when there are strong transition-transversion and G + C-content biases. Molecular Biology and Evolution 9: 678-687.

Thompson J.D., Higgins D.G., Gibson T.J., 1994. CLUSTAL W: improving the sensitivity of progres- 
sive multiple sequence alignment through sequence weighting, position-specific gap penalties and weight matrix choice. Nucleic Acids Research 22: 4673-4680.

Tibpromma S., Hyde K.D., McKenzie E.H., Bhat D.J., Phillips A.J.L., ... Karunarathna S.C., 2018. Fungal diversity notes 840-928: micro-fungi associated with Pandanaceae. Fungal Diversity 93: 13-160.

Úrbez-Torres J.R., 2011. The status of Botryosphaeriaceae species infecting grapevines. Phytopathologia Mediterranea 50: S5-S45.

Úrbez-Torres J.R., Gubler W.D., 2009. Pathogenicity of Botryosphaeriaceae species Isolated from Grapevine Cankers in California. Plant Disease 93: 584-592.

Úrbez-Torres J.R., Leavitt G.M., Voegel T.M., Gubler W.D., 2006. Identification and Distribution of Botryosphaeria spp. associated with grapevine cankers in California. Plant Disease 90: 1490-1503.

Úrbez-Torres J.R., Leavitt G.M., Guerrero J.C., Guevara J., Gubler W.D., 2008. Identification and pathogenicity of Lasiodiplodia theobromae and Diplodia seriata, the causal agents of bot canker disease of grapevines in Mexico. Plant Disease 92: 519-529.

Urbez-Torres J.R., Peduto F., Striegler R.K., Urrea-Romero K.E., Rupe J.C., ... Gubler W.D. 2012. Characterization of fungal pathogens associated with grapevine trunk diseases in Arkansas and Missouri. Fungal Diversity 52: 169-189.

Wagner D.B., Furnier G.R., Saghai-Maroof M.A., Williams SM, Dancik B.P., Allard R.W., 1987. Chloroplast DNA polymorphisms in lodgepole and jack pines and their hybrids. PNAS 84: 2097-2100.

Waite H., Armengol J., Billones-Baaijens R., Gramaje D., Hallen F., ... Smart R., 2018. A protocol for the management of grapevine rootstock mother vines to reduce latent infections by grapevine trunk pathogens in cuttings. Phytopathologia Mediterranea 57: 384-398.

White T.J., Bruns T., Lee S.J.W.T., Taylor J., 1990. Amplification and direct sequencing of fungal ribosomal RNA genes for phylogenetics. PCR protocols: a Guide to Methods and Applications 18: 315-322.

Zhang W., Groenewald, J.Z., Lombard, L., Schumacher, R.K., Phillips, A.J.L., and Crous, P.W. 2021. Evaluating species in Botryosphaeriales. Persoonia-Molecular Phylogeny and Evolution of Fungi 46: 63-115. 